\title{
Tuberculin reactivity in bacille calmette-guerin vaccinated individuals with sputum positive pulmonary tuberculosis
}

\author{
P.K. Jha ${ }^{1}$, R. Gurung ${ }^{2}$, N. Gyawali ${ }^{3}$, H.P. Nepal ${ }^{4}$, D.D. Baral ${ }^{5}$, S.K. Bhattacharya ${ }^{6}$ \\ ${ }^{1}$ Senior Demonstrator, ${ }^{2}$ Associate Professor, ${ }^{3}$ Post Graduate Student, ${ }^{6}$ Professor and Head, Department of Microbiology, B. P. \\ Koirala Institute of Health Sciences (BPKIHS), Dharan, Nepal, ${ }^{4}$ Lecturer, Department of Microbiology, Chitwan Medical \\ College, Bharatpur, Nepal, ${ }^{5}$ Assistant Professor and Director, Central Epidemiology Unit, BPKIHS, Dharan, Nepal
}

\begin{abstract}
The study was carried out to assess the value of tuberculin skin test for the diagnosis of Tuberculosis (TB) in BCG vaccinated individuals and to find out the sensitivity rate of tuberculin skin test (TST) in comparison to acid fast bacilli positive pulmonary TB. Similar population with high BCG vaccination coverage having both pulmonary TB patients $(\mathrm{n}=150)$ and apparently healthy individuals $(\mathrm{n}=150)$ in eastern Nepal were enrolled in this comparative study. Sputum from all the subjects was subjected to Z-N microscopy. TST was performed on these subjects by standard Mantoux method. Among the patients (mean age 36.18 \pm 14.15 yrs) and controls (mean age $35.61 \pm 13.44 \mathrm{yrs}$ ), $55.33 \%$ and $47.33 \%$ respectively have been found to be positive PPD reactors $(\geq 10 \mathrm{~mm})$ which is statistically not significant $(\mathrm{P}>0.05)$. About $23 \%$ of the patients $\& 27 \%$ of controls failed to react (anergic) to 5 TU PPD. PPD reactivity rate was high in the patients whose sputum smears were graded as 1+ in Z-N microscopy. The sensitivity of TST was highest for the induration of $\geq 5 \mathrm{~mm}(77.3 \%)$. Specificity increased with increasing size of induration and was highest $(73.3 \%)$ with induration of $\geq 15 \mathrm{~mm}$. Tuberculin skin test is an all or none phenomenon. In Nepal, tuberculin test has a limited value in the diagnosis of pulmonary TB as both the apparently healthy individuals and patients with sputum smear positive pulmonary TB who had been immunized with BCG, showed almost similar rate of tuberculin reactivity.
\end{abstract}

Key words: Tuberculin skin test, BCG vaccine, PPD tuberculin, Tuberculosis, Nepal.

\section{Introduction}

Tuberculin reaction is a delayed-type hypersensitivity (DTH) reaction which attempts to measure cell-mediated immunity (CMI) in the form of type IV immune response to the most commonly

Correspondence: P.K. Jha

E mail: j_pramod1@yahoo.com used purified protein derivative (PPD) of tuberculin ${ }^{1}$. When small quantities of antigen are injected intradermally, a hallmark response is elicited which includes induration, swelling and monocytic infiltration into the site of injection within 24-72 hours. ${ }^{2}$ Tuberculin skin test (TST) has an outstanding ability to distinguish 
P.K. Jha et al. Tuberculin reactivity in Bacille Calmette-Guerin (BCG).

Mycobacterium tuberculosis (M TB) infected persons from uninfected ones. A positive result indicates the presence of latent or active tuberculosis (TB) infection; a negative result does not rule it out.

The incidence of TB is high and is an important health problem in $\mathrm{Nepal}^{3}$. Each year, it is estimated that $77 / 100,000$ new smear positive cases are added to the existing TB patient population. ${ }^{4}$ As a part of control strategy, Bacillus Calmette Guerin (BCG) vaccination programme in Nepal was first introduced in 1966 and has been continued as an expanded programme on immunisation (EPI) since 1978/79. 5,6 BCG vaccination offers a greater protection against $\mathrm{TB}$, meningitis and disseminated TB but is ineffective against adult TB when given in infancy in high prevalence areas. ${ }^{7}$ Although the exact duration of its preventive effect is not known, it is possible that it may interfere with TST and leads to confusion in the diagnosis of TB. However, there is no reliable method for differentiation of reaction caused by vaccination, TB infection and infection due to non-tuberculous mycobacteria. ${ }^{8}$

We conducted this study to evaluate the usefulness of the TST in diagnosing pulmonary TB and to correlate the findings of TST with bacilloscopic findings and to calculate the predictive values of TST in BCG vaccinated subjects with pulmonary TB.

\section{Materials and methods}

All the patients who submitted their sputum specimens from August 2008 to July 2009 in TB research laboratory, BP Koirala Institute of Health Sciences (BPKIHS), suspected of TB were enrolled in this study. Subject selection and procedural outline have been depicted in the following flowchart (Figure 1).

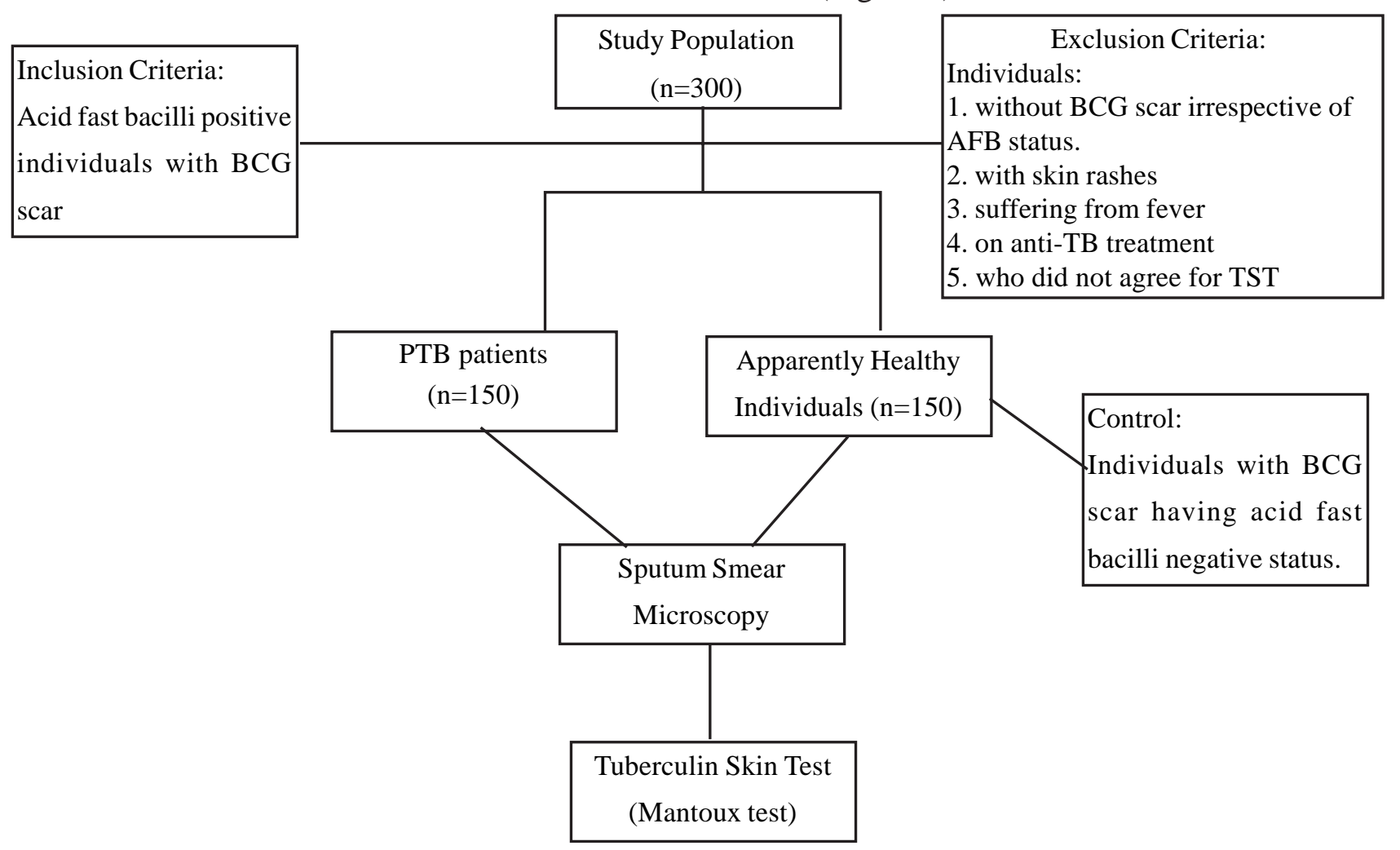

Figure 1: Subject selection and procedural outline 
Journal of College of Medical Sciences-Nepal,2011, Vol-7,No-1

For control, a group of apparently healthy individuals from indigenous population of Dharan municipality were recruited by random sampling method.

All the sputum specimens received in laboratory were subjected to Ziehl-Neelsen (Z-N) microscopy. Specimen collection, smear preparation, staining and reporting of the slides were done using standard guidelines developed by National TB Control Programme. ${ }^{9}$ A written informed consent was taken from all the patients and control individuals to perform TST. TST was performed by standard Mantoux method. ${ }^{10}$ A volume of $0.1 \mathrm{ml}$ of 5 TU PPD tuberculin was injected at angle of 5 to 15 degree intradermally on the lateral aspect of the flexor surface of the forearm 2-4 inches below the antecubital fossa. The result was read 48 to $72 \mathrm{hrs}$ after injection and the diameter of induration (in $\mathrm{mm}$ ) was measured by Sokal Ballpoint-Pen Method. ${ }^{11}$

A structured questionnaire was used to collect demographic information: age, weight, height, gender and residence; history of BCG vaccination and past TST; and history of TB and other infectious diseases.

Statistical analysis was performed using SPSS PC ${ }^{+}$ Version 12. For descriptive statistics, percentage, median and inter quartile range (IQR) were calculated whereas for inferential statistics nonparametric tests like chi-square $\left(\dot{\circ}^{2}\right)$ test, MannWhitney U test and Kruskal-Wallis test were used.

\section{Results}

Among 300 study population, 150 (94 males and 56 females) were new sputum smear positive pulmonary TB patients while 150 (63 males and 87 females) were controls. Approximately two third of the subjects were adults (16-49 yrs). The diameter of indurations in different age group is shown in Table 1.

A significant proportion $(\mathrm{P}=0.024)$ of the patients having 1+ (10-99/100 OIF) sputum AFB smear grading had diameter of indurations in the range of 1-9 $\mathrm{mm}$ and 10-15 $\mathrm{mm}$ (positive reaction).

Table 1: Diameter of indurations in different age groups.

\begin{tabular}{|c|c|c|c|c|c|c|c|}
\hline \multirow[t]{2}{*}{ Subjects } & \multirow[t]{2}{*}{ Sex } & \multirow[t]{2}{*}{ Age group } & & \multicolumn{3}{|c|}{ Diameter of indurations(mm) } & \multirow[t]{2}{*}{ P-Value } \\
\hline & & & 0 & $1-9$ & $10-15$ & $>15$ & \\
\hline & Male & $9-49$ & 2 & 26 & 29 & 14 & $0.001^{\mathrm{a}}$ \\
\hline & & $\geq 50$ & 9 & 4 & 9 & 1 & \\
\hline \multirow[t]{4}{*}{ Patient } & Female & $9-49$ & 2 & 14 & 18 & 3 & 0.101 \\
\hline & & $\geq 50$ & 6 & 4 & 8 & 1 & \\
\hline & Male & $9-49$ & 4 & 18 & 20 & 11 & 0.389 \\
\hline & & $\geq 50$ & 2 & 4 & 3 & 2 & \\
\hline \multirow[t]{2}{*}{ Control } & Female & $9-49$ & 27 & 10 & 18 & 7 & 0.441 \\
\hline & & $\geq 50$ & 8 & 6 & 7 & 4 & \\
\hline
\end{tabular}

$a=$ significant 
P.K. Jha et al. Tuberculin reactivity in Bacille Calmette-Guerin (BCG).

Correlation of TST indurations size with bacillary load is shown in figure 2.

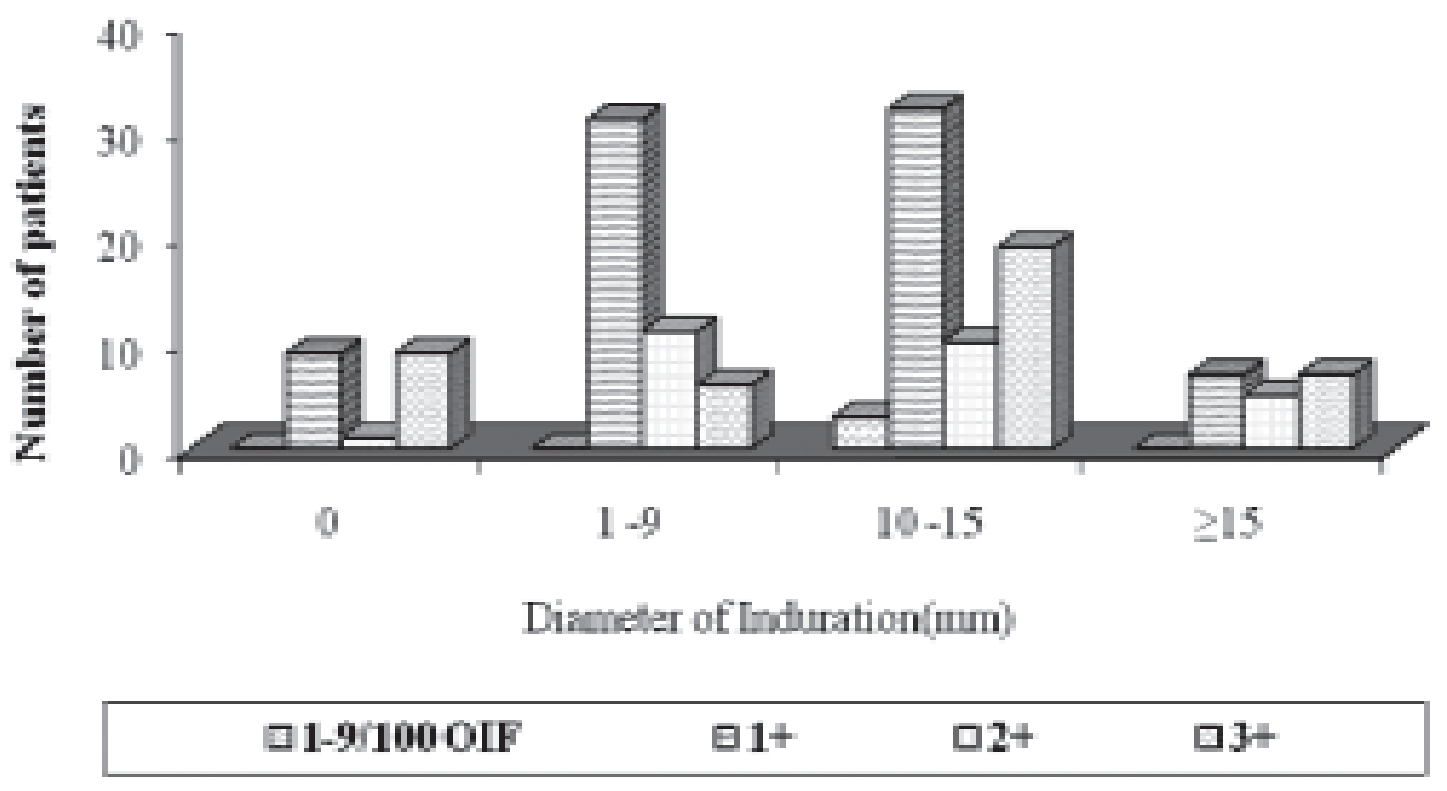

Figure 2: Bacilloscopic finding correlated with TST induration size among TB patients.

A significant proportion $(\mathrm{P}=0.024)$ of the patients having 1+ (10-99/100 OIF) sputum AFB smear grading had diameter of indurations in the range of 1-9 $\mathrm{mm}$ and $10-15 \mathrm{~mm}$ (positive reaction).
Comparison of PPD reactivity at different cut-off values between patients and controls was statistically not significant (table 2).

Table 2: Comparison of PPD reactivity at different cut-off values between patients and controls.

\begin{tabular}{ccccc}
\hline Diameter of Induration (mm) & Test result & Patients & Control & P-Value \\
\hline$\geq 5$ & Positive & 116 & 105 & 0.149 \\
& Negative & 34 & 45 & \\
& Positive & 83 & 71 & 0.165 \\
$\geq 10$ & Negative & 67 & 79 & \\
& Positive & 35 & 40 & 0.257 \\
& Negative & 115 & 110 & \\
Median (IQR) & $10(5-14)$ & $8(0-15)$ & & 0.265 \\
\hline
\end{tabular}


Journal of College of Medical Sciences-Nepal,2011, Vol-7,No-1

The sensitivity, specificity and predictive values of TST calculated at different cut off value is shown in table 3 .

Table 3: Sensitivity, specificity and predictive values of TST at different cut-off values.

\begin{tabular}{ccccc}
\hline $\begin{array}{c}\text { Diameter of } \\
\text { Indurations }(\mathrm{mm})\end{array}$ & $\begin{array}{c}\text { Sensitivity } \\
(\%)\end{array}$ & $\begin{array}{c}\text { Specificity } \\
(\%)\end{array}$ & Positive & Negative \\
\hline$\geq 5$ & 77.3 & 30.0 & 52.4 & 56.9 \\
$\geq 10$ & 55.3 & 52.6 & 53.8 & 54.1 \\
$\geq 15$ & 23.3 & 73.3 & 46.6 & 48.8 \\
\hline
\end{tabular}

The highest sensitivity (77.3\%) was found at cut off $\geq 5 \mathrm{~mm}$ whereas lowest sensitivity (23.3\%) and highest specificity $(73.3 \%)$ was at cut off $\geq 15 \mathrm{~mm}$.

\section{Discussion}

Tuberculin skin test has an outstanding ability to distinguish $M$. tuberculosis-infected from uninfected persons. After acquiring infection by inhaling aerosolized $M$. tuberculosis, the majority of individuals develop CMI which prevents progression to clinical TB. Such exposure also results in development of cutaneous sensitivity to PPD. ${ }^{12}$ Hence, the rationale of testing should be considered while interpreting the test result. The interpretation of TST in individuals vaccinated with $\mathrm{BCG}$ is of major significance in our population due to the high BCG vaccination coverage (88.1$96.7 \%) .{ }^{13}$

In this study, $55.33 \%$ of PTB patients and $47.33 \%$ of control subject have been found to be positive PPD $(\geq 10 \mathrm{~mm})$ reactors $(\mathrm{P}>0.05)$. A significant difference in PPD reactivity between patients with active TB and controls $(\mathrm{p}<0.05)$ was reported by Jamil et al. ${ }^{14}$
Miret-Cuadras et al, reported that $>30 \%$ of healthy individuals in high-prevalence countries had positive TST reaction. ${ }^{15}$ In accordance with the above study, the present study also revealed the high percentage $(47.33 \%)$ of tuberculin reactors with indurations of $\geq 10 \mathrm{~mm}$ among controls.

The sensitivity and specificity of Mantoux test has been found to be $67.5 \%$ and $60 \%$ respectively by Shaikh et al. ${ }^{16}$ The sensitivity of MT in our study patients was highest (77.3\%) for induration of $\geq 5 \mathrm{~mm}$ where as specificity increased with increasing size of induration and was highest (73.3\%) with induration of $\geq 15 \mathrm{~mm}$. The high TST positivity rates in our study may be a result of high prevalence of $\mathrm{TB}$, neonatal $\mathrm{BCG}$ vaccination superimposed on the immunity that develops following natural infection with increasing age and cross-reactions caused by the presence of atypical mycobacteria. However there are limited data regarding the incidence of atypical mycobacterial infection in Nepal. 
P.K. Jha et al. Tuberculin reactivity in Bacille Calmette-Guerin (BCG)......

It is usually acknowledged that BCG vaccination yields positive tuberculin reactions that, in general, have a smaller diameter and last for a shorter time than those caused by M TB infection. But there are no criteria, either for induration diameter or for the duration of sensitivity to tuberculin, that make it possible to differentiate, in all cases, a tuberculin reaction of vaccinal origin from one caused by $M$ TB infection. ${ }^{15}$ With special reference to tropical countries, it has been proposed that the high prevalence of exposure to other infections, including parasites, bias the immune system away from Th1 and DTH responsiveness or may compete for attention or space within the T lymphocyte compartment and thus reduces the DTH response to tuberculin induced by BCG over time. ${ }^{17}$

TST induration of $\geq 10 \mathrm{~mm}$ in majority of adults among controls, though found negative by ZiehlNeelsen microscopy of their sputum (the microscopy being positive only when the sputum contains $5000-10000$ bacilli/ml $),{ }^{9}$ may not imply that induration was due to BCG vaccination. As, the $\mathrm{BCG}$ vaccination programme in Nepal has been started long back (1978 AD), a possible waning period, it may signify that TST positivity in the adult population is related to natural infection rather than previous vaccination.

Though we did not determine the status of HIV infection, the spectrum of anergic to positive PPD reaction in a significant proportion of control subjects, on the other side of the coin, suggest that there could be a milieu for reactivation $\mathrm{TB}$ in this population especially in the presence of factors such as HIV infection, poverty, malnutrition, poor housing conditions etc. According to Brooks et al, $90 \%$ of persons with $10 \mathrm{~mm}$ indurations have $\mathrm{M}$ TB infection and the lifetime risk of reactivation TB is $20 \%$ or more among such persons. ${ }^{18}$

Plenty of evidence suggests that there is increased reactivation of latent $\mathrm{TB}$ and its increased transmission due to the immunodeficiency associated with HIV disease. There is high prevalence of HIV ( $5 \%$ cluster prevalence) in the eastern part of Nepal, though documented data is of $0.5 \% .{ }^{19}$ Nonetheless, whether or not a particularly infected person will become ill is impossible to predict with certainity. However, it has been estimated that $5 \%$ of infected persons get sick within 12-24 months of being infected; another $5 \%$ heal initially but, after years or decades, develop active TB either in the lungs or elsewhere in the body. ${ }^{19}$

A significant proportion (27.33\%) of controls was non-reactive to TST. This may indicate lesser probability of progression to active or inactive disease. Anergy to PPD is a common feature among HIV infected individuals and this does not preclude to developing the disease. Similar to the results of Nash and Douglass ${ }^{20}$ (25\%), we found $22.66 \%$ patients to be anergic. Magnani et al attributed the anergy to the M TB specific T cells that do not secrete interferon-gamma (IFN-ã) and lack the cutaneous lymphocyte antigen skin-selective homing receptor. ${ }^{21}$

The present study showed significant relationship between gender and size of induration. Males were more reactive than females. A similar result has 
been found by Roelsgaard et al. ${ }^{22}$ The male predominance in DTH response to tuberculin may be attributed to exposure of males to the external environment that may harbour M. TB or environmental mycobacteria.

Our study demonstrated a correlation between age and size of induration. The TST positivity was high among the age group <50 years whereas it was found to be low in the group $>50$ years. The progressive loss of tuberculin sensitivity with increasing age in the elderly remain obscured and the waning sensitivity could reflect general changes in the cellular immunologic mechanism, local factors in the ageing skin, or a gradual loss of reactivity with time after infection. This correlation of TST positivity and increasing age is also highlighted by Lienhardt et al. ${ }^{23}$

The present study revealed that $55.4 \%$ of patients having sputum smear positive for AFB were found to be positive PPD reactors ( $\geq 10 \mathrm{~mm})$, the finding being in accordance with that of Solangi $(55 \%) .^{24}$ Paranjape et al did not find any significant correlation between bacillary load in the sputum and parameters of CMI. ${ }^{25}$ We found that the PPD reactivity rate was significantly higher in those patients whose sputum smears were graded as $1+$. This signifies that the tuberculin reactivity does not exclusively depend on bacillary load but on several factors including virulence of the TB bacilli and immunogenetics of the individuals. ${ }^{26,27}$

\section{Conclusion}

This study emphasizes that the tuberculin reaction is an all or- none phenomenon. The TST does not reliably separate active $\mathrm{TB}$ infection from prior immunization with $\mathrm{BCG}$, latent infection or infection with environmental mycobacteria. In view of epidemiological circumstances in Nepal, TST has a limited value in the diagnosis of pulmonary TB since both the controls and patients with sputum smear positive pulmonary TB that had been immunized with BCG, revealed almost similar rate of tuberculin reactivity. Therefore tuberculin skin test should be requested only when the need for doing so is obviously clear in the physician's mind. Thus rational use of TST can reduce overdiagnosis.

\section{Acknowledgement}

We express our gratitude to all staffs of Tuberculosis laboratory and DOTS clinic, BPKIHS, Dharan for their generous help and co-operation in conducting this study.

\section{References}

1. U. Mack, G.B. Migliori, M. Sester, et al. LTBI: latent TB infection or lasting immune responses to M. TB? A TBNET consensus statement. Eur Respir J 2009; 33(5):956-73.

2. C.A. Black. Delayed type hypersensitivity: Current theories with an historic perspective. Dermatol Online $J$ 1999; 5(1): 7 .

3. C.T. Sreeramareddy, K.V. Panduru, S.C. Verma, et al. Comparison of pulmonary and extrapulmonary TB in Nepal- a hospital-based retrospective study. BMC Infect Dis 2008; 8:8.

4. WHO, Country Profile, Nepal, Available at: http:// www.who.int/GlobalAtlas/predefinedReports/TB/ PDF_Files/npl.pdf. Accessed August 10, 2009. 
5. H. Dixit. The Quest For Health: Disease that matter in Nepal, TB. $2^{\text {nd }}$ edi. Kathmandu: Educational enterprises (P) Ltd; 1999:116-7.

6. B.K. Suvedi. Immunisation programme of Nepal: An update. KUMJ 2003; 2(7): 238-43.

7. R. Sleiman, M. Al-Tannir, G. Dakdouki, et al. Interpretation of the tuberculin skin test in bacille Calmette-Guérin vaccinated and nonvaccinated school children. Pediatr Infect Dis J 2007; 26(2):134-8.

8. B. Ramin, D. Kam, B. Feleke, et al. Smoking, HIV and non-fatal TB in an urban African population. Int $J$ Tuberc Lung Dis 2008; 12(6):695-7.

9. A. Harries, D. Maher, M. Uplekar. National Tuberculosis Programme of Nepal: A Clinical Manual, National Tuberculosis Centre, $1^{\text {st }}$ edi. Thimi, Bhakatpur. 1998.

10. The WHO Standard Tuberculin Test. WHO/TB/Techn Guide/3;1963.

11. J.E. Sokal. Measurement of delayed skin-test responses. N Engl J Med 1975; 93:501-2.

12. R. Hussain, Z. Toossi, R. Hasan, et al. Immune response profile in patients with active TB in a BCG vaccinated area. Southeast Asian J Trop Med \& Public Health 1997; 28(4):764-73.

13. H. Shah. A cluster survey for determination of regular vaccination coverage among children. J Nepal Med Assoc 2008; 47(170): 91-3.

14. B. Jamil, S. Qamruddin, R.A. Sarwari, et al. An assessment of Mantoux test in the diagnosis of TB in a BCG-vaccinated, TB- Endemic area. Infect Dis J 2008; 17(1): 18-22.

15. P. Miret-Cuadras, J.M. Pina-Gutierrez, S. Juncosa. Tuberculin reactivity in Bacillus Calmette-Guerin vaccinated subjects. Tuber Lung Dis 1996; 77(1): 52-8.

16. A. Shaikh, K.M. Gurbakhshani, A. Jalbani, et al. Results of tuberculin test in AFB positive patients of TB compared with age and sex matched healthy controls. Med Channel 2005; 11(2): 78-81.

17. D. Elias, D. Wolday, H. Akuffo, et al. Effect of deworming on human $\mathrm{T}$ cell responses to mycobacterial antigens in helminth-exposed individuals before and after Bacille Calmette-Guerin (BCG) vaccination. Clin Exp Immun 2001;123(2): 219-25

18. G.F. Brooks, J.S. Butel, S.A. Morse, et al. Cases and clinical correlations. Jatwetz, Melnick and Adelberg's Medical Microbiology. 23rd edi. Singapore: McGrawHill Companies; 2004:734-70.

19. E.A. Asuquo, N.A. Dairo, L. Abia-Bassey, et al. Tuberculin skin test (TST) indurations in smear positive TB patients and healthy individuals in Calabar, Nigeria. Ethno-Med 2009; 3(1):75-9.

20. D.R. Nash, J.E. Douglass. Anergy in active TB. A comparison between positive and negative reactors and an evaluation of $5 \mathrm{TU}$ and $250 \mathrm{TU}$ skin test doses. CHEST 1980;77(1):32-7.

21. Z.I. Magnani, C. Confetti, G. Besozzi, et al. Circulating, Mycobacterium TB-specific lymphocytes from PPD skin test-negative patients with TB do not secrete interferon-gamma (IFN-ã) and lack the cutaneous lymphocyte antigen skin-selective homing receptor. Clin Exp Immunol 2000;119(1):99-106.

22. E. Roelsgaard, E. Iversen, C. Blocher. TB in tropical Africa: an epidemiological study. Bull World Health Organ 1964;30:459-518.

23. C. Lienhardt, K. Fielding, J. Sillah, et al. Risk Factors for TB Infection in Sub-Saharan Africa. A contact study in the Gambia. Am J Respir Crit Care Med 2003; 168(4): 448-55.

24. A.R. Solangi. Comparative assessment of clinical, radiological, sputum smear and Mantoux test for diagnosis of pulmonary TB. Chest Meeting Abstracts 2008; 134:155003.

25. R.S. Paranjape, A. Ravoof, G.S. Acharyulu, et al. Cell mediated immune response in south Indian pulmonary TB patients. Indian J Tuberc 1988; 35(4): 63-70.

26. J. Dormans, M. Burger, D. Aguilar, et al. Correlation of virulence, lung pathology, bacterial load and delayed type hypersensitivity responses after infection with different Mycobacterium TB genotypes in a BALB/c mouse model. Clin Exp Immunol 2004; 137(3):460-8.

27. Z. Toossi, J.J. Ellner. Pathogenesis of TB: immunogenetics of TB. Friedman LN. TB: current concepts and treatment. 2nd edi. New York: CRC Press; 2001: 26-7. 